International Journal of Canadian Studies

Revue internationale d'études canadiennes

INTERNATIONAL JOURNAL

\title{
L'impact de la radiophonie commerciale sur la poésie vocale québécoise
}

\author{
Jean-Nicolas De Surmont
}

Numéro 39-40, 2009

Culture - Natures in Canada

Culture - natures au Canada

URI : https://id.erudit.org/iderudit/040823ar

DOI : https://doi.org/10.7202/040823ar

Aller au sommaire du numéro

\section{Éditeur(s)}

Conseil international d'études canadiennes

\section{ISSN}

1180-3991 (imprimé)

1923-5291 (numérique)

\section{Découvrir la revue}

\section{Citer cet article}

De Surmont, J.-N. (2009). L'impact de la radiophonie commerciale sur la poésie vocale québécoise. International Journal of Canadian Studies / Revue internationale d'études canadiennes, (39-40), 63-81.

https://doi.org/10.7202/040823ar

\section{Résumé de l'article}

Dans un article précédent (De Surmont, 2001) nous avons mis en relief le faitqu'avec l'arrivée de l'industrie et des médias de masse, lacarrière de chanteur s'est transformée en celle de médiateur,c'est-à-dire d'un agent interagissant au sein de l'activitéchansonnière mais qui occupera aussi une place au sein de la presse et du champlittéraire. Ainsi, certains chanteurs, paroliers ou poètes, dont les poésies sontmises en chansons ou en musique, sont parfois éditeurs de journaux (Napoléon Aubin)ou typographes (Roch Lettoré); d'où la tradition del'auteur-éditeur-imprimeur. Il conviendrait d'évoquer la fonctionmultiple du journaliste durant la période qui va de l'èclosion de la pressecanadienne-française jusqu'à la fin du XIX ${ }^{\mathrm{e}}$ siècle. Parla suite, c'est la radio et le phonogramme qui deviendront, au début duXX ${ }^{\mathrm{e}}$ siècle, les supports privilégiés de la diffusion del'objet-chanson favorisant comme d'autres inventions la circulationdes idées; pensons au télégraphe également. Dans cet article, nous mettrons enrelation l'essor de la radiophonie et celui de la chanson signée et nousmontrerons les corrélations entre les fonctions de la radio et les conditionshistoriques. En quoi les confronter nous permet-il de mieux connaître les contextesfavorables à la naissance et à l'évolution de la radio et à la diffusiond'une culture populaire? Telle est une question qui sous-tend notre travail.L'étude que nous proposons concerne principalement la dimensionsocio-historique de la radiophonie et son rôle de canalisation dans la carrière desinterprètes ou auteurs-compositeurs de la chanson québécoise (nous ne pouvonsélargir notre propos à l'ensemble des artisans de la chanson comme lesresponsables de tournée, agents, producteurs, etc.). Plus généralement nousétudierons la démocratisation de la culture par la radiophonie.
Tous droits réservés @ Conseil international d'études canadiennes, 2009
Ce document est protégé par la loi sur le droit d'auteur. L'utilisation des services d'Érudit (y compris la reproduction) est assujettie à sa politique d'utilisation que vous pouvez consulter en ligne.

https://apropos.erudit.org/fr/usagers/politique-dutilisation/ 


\title{
Jean-Nicolas De Surmont
}

\section{L'impact de la radiophonie commerciale sur la poésie vocale québécoise}

\section{Résumé}

Dans un article précédent (De Surmont, 2001) nous avons mis en relief le fait qu'avec l'arrivée de l'industrie et des médias de masse, la carrière de chanteur s'est transformée en celle de médiateur, c'est-à-dire d'un agent interagissant au sein de l'activité chansonnière mais qui occuperà aussi une place au sein de la presse et du champ litteraire. Ainsi, certains chanteurs, paroliers ou poètes, dont les poésies sont mises en chansons ou en musique, sont parfois éditeurs de journaux (Napoléon Aubin) ou typographes (Roch Lettoré); d'où la tradition de l'auteur-éditeur-imprimeur. Il conviendrait d'évoquer la fonction multiple du journaliste durant la période qui va de l'éclosion de la presse canadienne-française jusqu'à la fin du XIX $X^{e}$ sièclel. Par la suite, c'est la radio et le phonogramme qui deviendront, au début du $X X^{e}$ siècle, les supports privilégiés de la diffusion de l'objet-chanson favorisant comme d'autres inventions la circulation des idées; pensons au télégraphe également. Dans cet article, nous mettrons en relation l'essor de la radiophonie et celui de la chanson signée et nous montrerons les corrélations entre les fonctions de la radio et les conditions historiques. En quoi les confronter nous permet-il de mieux connaître les contextes favorables à la naissance et à l'évolution de la radio et à la diffusion d'une culture populaire? Telle est une question qui sous-tend notre travail. L'étude que nous proposons concerne principalement la dimension socio-historique de la radiophonie et son rôle de canalisation dans la carrière des interprètes ou auteurs-compositeurs de la chanson québécoise (nous ne pouvons élargir notre propos à l'ensemble des artisans de la chanson comme les responsables de tournée, agents, producteurs, etc.). Plus généralement nous étudierons la démocratisation de la culture par la radiophonie.

\begin{abstract}
In a previous article (De Surmont, 2001), we highlighted how the arrival of industry and mass media transformed the singing career into one of mediator, an interacting agent of song writing that also had a place in the press, in literature, etc. Certain singers, songwriters, or poets whose poetry was put to song or to music, were also sometimes editors (Napoléon Aubin), or printers (Roch Lettoré), hence the tradition of the author-editor-printer. Consider also the multiple functions of the journalist during the period beginning with the coming into being of the French Canadian press through the end of the nineteenth century. I Afterwards, in the early twentieth century, radio and sound recording became the privileged platforms of the song-as-object ('objet-chanson) which, like the telegraph and other inventions, favoured the circulation of ideas. In this article, we juxtapose the development of radio
\end{abstract}


with the original song composition (chanson signee), and show correlations between historical conditions and the function of radio. Our aim is to better understand those contexts that favoured the birth and evolution of radio itself as well as the broadcasting of a popular culture. The study focuses mainly on the socio-historical dimension of radio and its role in steering the careers of singers or songwriters of the chanson québécoise (our analysis could not include all artisans in the music industry such as tour managers, agents, producers, etc.). More generally, we look at the democratization of culture through radio.

\section{État de la question}

Ces dernières décennies, l'histoire de la chanson québécoise a fait l'objet de plusieurs études. Parallèlement, l'histoire de la discographie québécoise a aussi attiré l'attention de chercheurs comme Jean-Jacques Schira, Robert Thérien et Danielle Tremblay. À part Robert Saucier et Robert SaintAmour qui ont écrit sur la programmation musicale radiophonique, peu d'auteurs se şont intéressés au rôle joué par les médias et plus spécialement par la radio dans la promotion de la chanson québécoise.

\section{La croissance démographique et l'industrie radiophonique}

Le développement de l'activité radiophonique va profiter de la croissance démographique des grandes villes. Le Québec connaît une croissance importante dans les premières décennies du siècle. Le nombre de villes de 10000 habitants et plus passe de 5 en 1901 à 8 en 1911 et à 18 en 1930. La ville de Montréal, où se situent les grandes avancées industrielles qui vont marquer l'essor de la radio, est peuplée de 618506 habitants en 1921 (Lamonde 18). A la fin des années 1920, il y a 75 stations de langue anglaise et française au Canada surtout situées dans les grandes villes, laissant ainsi non couverts les villages. Yvan Lamonde écrit à ce sujet :

En milieu urbain, le changement culturel qui s'impose repose sur des moyens de communication et de transmission (la publicité, l'automobile, l'électricité, le téléphone, la TSF), sur des supports inédits (le disque et la pellicule) et sur l'hégémonie nouvelle du monde du spectacle (le cinéma, le sport, le burlesque, le vaudeville, le théâtre, l'opéra, l'opérette) tant décrié par [Olivar] Asselin. (115)

S'ajoute également à ce problème le fait que plusieurs auditeurs canadiens syntonisaient les stations américaines dont l'activité de divertissement était plus réputée au détriment de l'aspect éducationnel et culturel que prendront bon nombre de stations canadiennes.

\section{Le rôle de Marconi}

La dimension éducative de la radiophonie ne doit pas faire oublier que le premier public que vise Marconi de la Canadian Marconi Company 
(Marconi Wireless Company of Canada) ${ }^{2}$ avec la CFCF était composé des classes moyennes et supérieures, ce qui semble aller de pair avec la croissance économique des villes et le fait que le Québec devient majoritairement urbain vers 1915. Dès les débuts de l'activité radiophonique canadienne le problème du financement des émissions se posa et Marconi dû suggérer l'insertion de messages publicitaires pour ponctuer les émissions ${ }^{3}$. Cela favorisera, à la fin des années 1920 , l'essor de la radiophonie éducative et commerciale, et par le fait même, la démocratisation de la radio. Il est normal qu'une société qui reconnaît le droit au loisir et à la diminution de la durée des heures de travail, voit se mettre en place une structuration du temps libre. La radio devint ainsi le moteur de l'orientation des goûts musicaux ou du moins de la diffusion du répertoire. Ainsi,

[a]u cours du $\mathrm{XX}^{\mathrm{e}}$ siècle, les compositeurs délaissent progressivement la formation symphonique développée au cours du siècle précédent-une évolution facilitée par les commandes radiophoniques. En favorisant la diffusion de certains genres musicaux, les radios jouent aussi un rôle déterminant dans l'évolution de l'art musical contemporain et [...] dans les politiques culturelles, si ce n'est dans la politique tout court. (L'Écuyer 959)

La radio, bien avant la création du réseau de Radio-Canada, devint un facteur de cohésion, non seulement parce que l'écoute dans les campagnes était collective, mais parce qu'elle rejoignait les objectifs du gouvernement fédéral. Ainsi, le Jubilé de 1927 commémorant la Confédération de 1867 est transmis en direct depuis Ottawa, touchant un public évalué à cinq millions d'auditeurs dans le monde entier (Cupido 112). Il s'agit du premier événement que le gouvernement fédéral utilisa pour inculquer des valeurs comme la citoyenneté démocratique et l'unité politique à travers tout le Canada. Plusieurs politiciens comme Mackenzie King et Lomer Gouin y prononcèrent leur discours. Des interprètes y chantèrent des chansons et hymnes comme le «O Canada », « Maple Leaf Forever » et " God Save the King ». Il s'agissait donc de chansons d'expression française (Charles Marchand s'y produisit dans la soirée) et anglaise que l'on connaissait tout aussi bien dans le public qu'à la Chambre des Communes (Cupido 112).

\section{Enregistrement sonore et programmation radiophonique}

Avec l'arrivée du disque, mais surtout avec la disparition de l'enregistrement acoustique au profit de l'enregistrement électrique en 1925, la radio va entraîner une modification de l'objet musical. On fait encore tourner des disques à la radio mais la qualité de ceux qui y sont diffusés s'améliore et les techniques en radio ont eu une influence sur l'enregistrement comme tel. Les nouvelles techniques d'enregistrement améliorent la qualité sonore des disques et favorisent l'enregistrement de pièces symphoniques dont l'enregistrement acoustique rendait 
difficilement les qualités. Ceci pourrait expliquer que même les versions imprimées dans les musiques en feuilles ou dans les recueils de chansons ne contiennent pas l'ensemble des strophes du texte chansonnier. La radio modifie pour ainsi dire la manière d'interpréter la chanson. À ce propos Luc Bellemare écrit:

Alors que la radio permet de rejoindre un auditoire plus éloigné, qu'elle perturbe le mode de réception du produit culturel et qu'elle suscite par la même occasion la création d'une image médiatisée plus forte, le disque 78 tours commence déjà à influer sur la manière d'interpréter de Marchand et des autres interprètes en général, au milieu des années 1920 . Plus encore la radio et le disque entraînent éventuellement chez l'auditoire une passivité contemplative qui s'en remet ainsi progressivement aux interprètes et diminue de façon significative sa participation active au renouvellement des chansons de tradition orale. (112)

Outre son facteur cohésif, la radio permet de perpétuer les pratiques chansonnières de la tradition orale. L'apparition de la radio s'inscrit dans un contexte plus large du passage de la transmission orale du corpus de la tradition orale (comme mode dominant de transmission du répertoire chansonnier) à la transmission médiatisée par la technique. Ainsi la transmission par l'interprète artisan laisse place à la transmission médiatisée, passant parfois, et même de plus en plus, par l'écrit et l'interprétation médiatisée (De Surmont, Les interactions). Il n'en demeure pas moins que la frontière est ténue entre la tradition du mot transcrit et parfois glosé et la parole enregistrée. La musique country, très influencée par la musique de tradition orale, a consisté en une sorte de passage esthétique favorisant la disparition progressive de la tradition orale en contexte familial et rural au profit de l'arrivée de la chanson américaine. Ainsi, les enregistrements de chansons signées et de monologues par des Canadiens français des années 1920 (par exemple J.-Hervey Germain, plus tard Mary Travers dite La Bolduc) font état de traits de prononciation comme on les trouvait encore dans les zones urbaines fortement anglicisées (comme Montréal) et rurales à l'époque.

La naissance de la radio ${ }^{4}$ coïncide aussi avec une forte mise en scène de la tradition orale (hors de son contexte d'origine) inaugurée par les veillées du bon vieux temps à Montréal en 1919 qu'organisent Marius Barbeau et Edouard-Zotique Massicotte à la Bibliothèque Saint-Sulpice, rue Saint-Denis à Montréal. De concert avec le microphone, la radio va favoriser l'essor des chanteurs de charme québécois. En effet, l'esthétique des chanteurs de charme à la voix feutrée et modulable est directement liéeà l'apparition du microphone et à celle de la radio, même si c'est surtout dans les années 1940 que ce mouvement va se répandre (c'est le rôle de la technologie comme élément constitutif de la texture sonore ou du discours musical). En effet, la technique et le style du crooning associés au développement du microphone dans les années 1920 et 1930 seront d'abord 
utilisés dans le cadre de la diffusion radiophonique avant de l'être dans le cadre de concerts. "Lionel Parent annonce, avec Jean Lalonde (1914-1991), la naissance des "crooners" québécois, des interprètes qui savent utiliser le microphone pour créer un climat intimiste qui plaît au public radiophonique » (Thérien 165). Avant cela les chanteurs ${ }^{5}$ devaient considérablement faire porter leur voix en abaissant le larynx de la même manière que les chanteurs classiques afin d'amplifier le volume sonore. Le métier d'ingénieur du son changea notablement dès lors qu'il ne fut plus obligatoire de s'époumoner devant un cornet pour créer suffisamment de volume sonore pour enregistrer.

De plus en plus, dans les années 1930, la radio intégrait la chanson dans ses émissions culturelles montrant ainsi le rôle de complice qu'elle jouait parfois avec le clergé qui reconnaissait en elle sa valeur éducative, artistique et religieuse. Par conséquent, la radio comportait non seulement une composante commerciale mais également une composante éducative et thématique dans plusieurs domaines (éducation religieuse, apprentissage de l'histoire du Canada, apprentissage du patrimoine vocal ancestral, etc.). La commercialisation de l'activité radiophonique va impliquer l'introduction de la format radio, c'est-à-dire une programmation axée sur « une cible homogène d'auditeurs partageant les mêmes goûts et les mêmes habitudes de consommation, auxquels il ne faut pas laisser le temps de s'ennuyer ou de chercher une autre station " (Fabbri 684). L'arrivée de la radio va contribuer à la création du phénomène de star comme l'indiquent David Bucley et John Shepperd :

With the advent of radio in particular, audiences for a particular performer or piece of music became significantly larger and more greatly dispersed. Although 'star performers' such as AI Jolson had undoubtedly existed before the age of radio, recording and film created a greater distance between performers and their mass audiences, and thereby gave rise to a space within which it was easier to construct the persona of the star. [...] The new media of radio, film and subsequently, television, together with innovation such as the phonograph and the increasing importance of Muzak, meant that taste communities could be created in both public and private spaces (in the bedroom, the workplace or even the supermarket), away from any direct experience of the performance itself ${ }^{6} .(160)$

\section{La culture à la radio : l'exemple de CKAC}

Si l'enregistrement sonore a délimité la durée des chansons et des pièces en restreignant ainsi les conditions de présentation de certaines formes musicales comme des extraits d'opérettes plutôt que des symphonies entières, de leur côté, la radio et le film vont favoriser le phénomène de la starification des chanteurs. Tous ces exemples ne sauraient rendre compte à eux seuls de l'importance que joua la radio dans les champs culturel, 
scientifique et politique dès ses débuts, notamment par le biais de CKAC sous la houlette du journaliste Jacques-Narcisse Cartier. Ainsi le poste privé CKAC ( Canada Kilocycle:Amerịca Canada »), propriété du journal de tendance libérale La Presse, eut pendant un certain temps son propre orchestre que dirigeaient des musiciens comme Henri Miro, Decellier et Edmond Trudel ${ }^{7}$ (Potvin 663). Cette première station francophone en Amérique est inaugurée le 27 septembre $1922^{8}$ par le pianiste Emiliano Renaud qui propose, à partir de 1925, la première émission d'enseignement en diffusant un cours de piano de trente semaines (Martel 63). La station CKAC bénéficie aussi de la compétence de Leonard Spencer dont on dit qu'il est « le premier technicien de la radio au Canada »(Du Berget et al. 19). Miro, aussi directeur à la maison Berliner de 1916. à 1921, prête régulièrement son concours comme compositeur à des chanteurs, témoignant ainsi du rapprochement du savant et du populaire à cette époque. La Gaspésienne Mary Travers dite La Bolduc, de même que Jacques Aubert s'y produisent. Entre autres, Fernand Perron dit le « Merle Rouge " y chante dès 1927 et se produit toujours à l'émission de la Living room furniture en 1933, commanditaire de l'émission d'Hector Pellerin (Benoit 70). L'émission du même titre du violoneux Isodore Soucy est diffusée pendant quatre ans à partir de 1926 sur les ondes de CKAC (Du Berger 145). Fernand Perron bénéficiera ainsi de son expérience radio avant d'enregistrer ses premiers disques. Le chanteur traditionaliste Charles Marchand (De Surmont, Dictionnaire bibliographique) se produit à CKAC à la fin des années 1920 lors de l'émission l'Heure Frontenac produite à partir de 1928 (Du Berger 145). La programmation des débuts de la radio est partagée entre la présentation de musique en direct, comme ce sera le cas principalement à CKAC jusqu'en 1927 (Baulu), et de musique enregistrée.

La station CKAC jouera un rôle important dans le cadre des croisades en faveur de la chanson française. L'influence du répertoire de la chanson française jumelée à l'écoute de la diction française par les animateurs de radio (pensons à Roger Baulu dans les années 1940) va profondément orienter le modèle traditionnel de prononciation, hérité du XVIIIe siècle, qui prévalait dans la population québécoise. Avec la radio, ce n'est plus seulement l'élite qui découvre un autre modèle de prononciation mais la population québécoise en général. Jean-Denis Gendron affirme à cet égard :

Cet autre modèle, les francophones du Canada ont appris à le connaître avec l'avènement dans les années 1920 de la radio et de ses speakers français ou parlant à la française, puis du cinéma parlant français dans les années 1930 et, enfin, de la télévision et des voyages de groupe en France, à partir des années 1950. Avec la Révolution tranquille des années 1960, les temps étaient mûrs pour que soient mis en branle, au sein de la société québécoise, les changements qui ont porté à l'avant-scène, grâce surtout à la radio 
et à la télévision, un nouveau modèle de prononciation: (Gendron 185)

La présence en direct de ces chanteurs sur les ondes radiophoniques vient en fait combler la piètre qualité de l'enregistrement sonore et le coût élevé des disques. Le discographe Edward Moogk explique ainsi la concurrence qui s'établit entre le disque et la radio :

La radio était dans la bonne voie. Le disque, malgré ses qualités, était incapable de soutenir la concurrence. De 1904 à 1925, l'industrie du phonographe n'avait pas réellement fait de progrès. L'enregistrement sur disque ignorait les hautes et les basses fréquences. De plus, tout imparfait que fût le son de la radio, il offrait à l'auditeur un registre plus étendu que le meilleur des disques. En piétinant sur place, l'industrie du disque avait manifestement désillusionné le public. (95)

Robert Saint-Amour affirme, à propos du rôle de la chanson :

$\mathrm{La}$ radio avait constitué une sorte de "libensraum" sonore, c'est-à-dire un espace vital sonore sans lequel la chanson québécoise n'aurait probablement pas connu l'essor qui fut le sien. Entre autres nombreux exemples, qu'on pense au rôle qu'avait tenu le poste de CKAC à ses tout premiers débuts, dans la carrière de La Bolduc; qu'on se souvienne de l'influence exercée sur les jeunes auteurs par Radio-Canada et son concours La chanson canadienne à la fin des années cinquante. Autant la radio avait été un lieu de continuité et d'unité par sa contribution à l'éclosion et à la diffusion d'une chanson plus authentiquement québécoise et non plus simplement folklorique, autant, m'apparaissait-il intuitivement, cette même radio menaçait la chanson en instaurant un degré extrême de discontinuité et de diversité de l'organisation spatiale. (87-88)

\section{Radiophonie et enregistrement sonore}

La radio devient plutôt un tremplin pour de nombreux musiciens de tradition orale qui, dans les années 1920, produisent leur émission ou se produisent en direct, alors que l'industrie du disque connaît au contraire un déclin des ventes. Encore à la fin des années 1940, la musique traditionnelle jouit d'une certaine popularité, par exemple à CHRC où est réalisée l'émission les Montagnards Laurentiens à laquelle participent, entre autres, Jos Bouchard et Théodore Duguay (Labbé 37), etc. Il semble clair que l'accès à une carrière radiophonique ne fut pas toujours le fruit d'une longue expérience et de longues études, mais s'improvisait souvent à tel point que l'on peut affirmer que la carrière radiophonique ne s'envisage pas comme une fin en soi sinon un moyen.

La radio permet la diffusion de récitals en direct, se substituant aux studios et aux salles de concerts. En outre, on enregistre parfois les 
émissions de radio, et de récentes inventions comme le gramophone en permettent la diffusion. En 1920, bien que près de 250000 personnes possèdent un gramophone (Anonyme 236), l'industrie de l'enregistrement de chansons de tradition orale n'en est qu'à ses débuts. Compo est la première compagnie canadienne à produire des disques enregistrés avec un microphone (1925) et à partir d'émissions de radio (1925) (Thérien 123). La chanson de tradition orale devra attendre la multiplication des récepteurs et, surtout, la baisse des prix du disque et l'amélioration de l'enregistrement sonore pour connaître un certain essor commercial. On perçoit parfois la radio comme un obstacle à la performance d'artistes sur scène. En revanche, elle fait aussi bénéficier les premiers chanteurs de charme d'un circuit de diffusion plus sûr que l'enregistrement sonore, encore tourné vers les productions américaines et françaises. En effet, «[a]u cours des années qui ont précédé la Seconde Guerre Mondiale, les disques à CKAC venaient presque en totalité de France et c'est surtout par le disque que le Canada-français a connu Alibert [...], Jean Sablon, Tino Rossi, [etc.] " (Baulu 61).

La radio et le disque joueront ce rôle fondamental dans l'histoire de la réception de la chanson et de la musique en général. Cela permet aux Canadiens d'entendre les compositeurs diriger eux-mêmes leurs pièces ou sinon des interprètes formés par le compositeur lui-même. Giuseppe De Luca affirme à cet égard : "When we can hear, .via the phonograph, the creator of Falstaff and Otello, we know that we are experiencing interpretations immortal in their concepts because they were formed under the rigourous supervision of the exacting composer himself $\eta^{9}$ (De Luca 9). Pourtant, peu de compositeurs du début du siècle enregistrent, au niveau mondial, leurs propres oeuvres. Notons Richard Strauss, Ignacy Paderewski, Sergei Rachmaninov et Arturo Toscanini (Bauer). Sinon, de façon générale, les artistes européens enregistrent les œuvres de leurs pays.

\section{L'âge d'or de la radio (1937-1953)}

Selon l'historien trop oublié Elzéar Lavoie, il faut en fait attendre 1937 pour que la radio connaisse véritablement sa croissance, alors que le taux de radiophonie atteint déjà $57 \%$. Notons la création dans les premières années radiophoniques de CBF à Montréal (1936), de CBV à Québec (1938), de CBJ à Chicoutimi (1938), de CKCV (1924) et de CHRC à Québec (1926), de CHMI à Chicoutimi (1928), de CHLP à Montréal et de CHNC à New Carlisle (1932), de CHLN à Trois-Rivières, de CHLT à Sherbrooke et de CJBR à Rimouski en 1937 (qui permet aux gens de Charlevoix de syntoniser Radio-Canada). Mentionnons aussi la multiplication de stations fantômes du CN comme la CNRM à Montréal (1925), CNRQ (1928) à Québec et des stations de courte durée comme celle du journal le Soleil, $\mathrm{CKCI}$, premier poste de radio de langue française à Québec, inauguré le 23 
mars 1924, et les stations CHJC opérant de 1922 à 1925 et CHCD de 1923 à 1925 (Du Berger, La Radio 27 et 29).

Certaines stations se spécialiseront dans un créneau d'information et de musique traditionnelle. CHRC, « était captée dans les campagnes, dans la Beauce, un peu partout. Il s'intéressait aux affaires des fermiers, proposait des recettes, présentait le chapelet à 7 heures, en somme, il donnait l'image d'un poste familial et traditionnel » (37). Le poste radiophonique CHRC confiera à Arthur Maheux, l'un des membres de la Société du Parler français au Canada, le soin de faire une chronique hebdomadaire d'épuration linguistique entre 1941 et 1943. Ces chroniques sont presque entièrement dévolues aux programmations de la chaîne de Radio-Canada dans les décennies qui suivront. Bien que CHRC soit la station où Félix Leclerc fait ses débuts à la radio en 1934, elle ne jouera pas le rôle essentiel comme CKVL à Verdun, dans la diffusion de la chanson française et canadienne. Radio d'abord bilingue avant de ne diffuser qu'en français, fondée en 1946, CKVL aurait permis, selon Jacques Normand, grâce à l'émission La parade de la chansonnette française (animée par Jacques Normand et Guy Maufette) :

a) « le relancement de la chanson populaire française;

b) la création de toutes pièces de vedettes inconnues;

c) un palmarès de chanson populaire [...];

d) la reconnaissance par les Français d'un nouveau débouché;

e) le début de carrières internationales pour les Canadiens (Québécois) : Félix Leclerc [qui y chante des chansons dès 1937], Blanchet, Raymond Lévesque, Ferland, Guylaine Guy...; [et]

f) un nouveau départ, une nouvelle politique pour les postes de radio $\gg .(57)^{10}$

Les conséquences de l'apparition de la radio de divertissement vont de pair avec l'essor du show-biz et des tournées d'artistes comme Madame Bolduc et Charles Marchand dans les années 1930. Le star system a définitivement contribué à façonner des vedettes locales autour de la Deuxième Guerre mondiale. La radio va considérablement élargir le cycle de production, de diffusion et de réception des phénomènes chansonniers. Depuis le début du siècle, on avait connu les débuts sur la scène de nombreux interprètes (Henri Cartal, Damase Dubuisson, Madame Bolduc, Alys Roby) mais la radio va élargir l'auditoire des chanteurs et créer le phénomène des stars. Plus tard, avec l'apparition de la télévision, certains vont diversifier leurs activités. Le cycle de diffusion des chansons (de tradition orale ou signées) évolue en fonction des différentes inventions issues du développement des télécommunications, lui-même le produit de la rationalité technique. Il correspond à une évolution qui peut varier selon la décennie ou même l'artiste en question. Leclerc, par exemple, fera ses 
débuts à la radio (Du Berger 70) et par la suite sur la scène, découvert par le radio-crochettiste Jacques Cannetti, avant d'endisquer.

Le développement de la radiophonie provoque aussi un déplacement des fêtes collectives et les gens « au lieu de sortir pour aller à un rassemblement, ont appris à rester chez eux, où le divertissement les rejoignait »(Pagé 15). Pendantl'entre-deux-guerres, le théâtre est supplanté par le radiothéâtre; la première création radiothéâtrale remonte à 1933. Les premières œuvres originales écrites pour la radio : le radioroman, mélodrame diffusé en plusieurs épisodes chaque semaine; la comédie de mœurs; les sketches ou causeries comiques sur la vie sociale, conçus sous forme de cabaret, de variétés ou de revue (Nielsen 105). Bien après avoir perdu sa fonction sociale, la chanson de tradition orale attirera de nouveau des spectateurs dans les salles d'émissions de variétés déplaçant de nouveau le mode de diffusion.

Les années 1930 à 1950 voient naître la haute fidélité en 1944, le microsillon ( 33 et 45 tours), qui remplace le 78 tours au début des années 1950 (le microsillon apparaît en 1947) et la stéréophonie. Tous ces progrès techniques permettent une meilleure qualité d'écoute et facilitent la commercialisation de la chanson. La bataille des vitesses de disque opposent le 33 tours à ses prédécesseurs, le 45 tours et le 78 tours. Au début des années 1950, le 45 tours est choisi pour être le support des chansons de consommation et les 33 tours pour les musiques de toutes sortes.

À la même époque, et avant l'apparition de la télévision, la radio connaît son âge d'or. L'avènement de la radio commerciale verra la relation étroite entre programmation et agences de publicité. Tout au long de son histoire, l'élaboration de la programmation radiophonique montrera d'étroits liens avec les habitudes d'écoute (savamment étudiées par les sondages BBM au Canada) et de consommation. Il y a aussi l'explosion des radios.

Dans les années 1950, avec le développement du secteur des radios privées, la musique commerciale est privilégiée sur les ondes radiophoniques. On compte alors environ 800 chaînes de radio en Amérique du Nord (Nielsen 96). La radio, et surtout la télévision qui commence à supplanter la radio à partir de 1953, modifient profondément l'activité chansonnière, comme nous l'avons expliqué, non seulement avec l'apparition des stars mais parce qu'ils offrent un territoire de diffusion plus important. Ces nouveaux médias sont rapidement associés au mode privilégié de diffuision de la variété, permettant la pénétration de plus en plus profonde en zone rurale de ce genre de musique aux dépens de la chanson de tradition orale. Ce mode de diffusion permet la fabrication et la promotion de vedettes :

a) Le chanteur et les artistes en général défilent pour interpréter leurs pièces. 
b) Des concours sont créés et relèvent des artistes (dans la tradition française dont fait partie l'imprésario Jacques Canetti).

c) Des chanteurs ou chansonniers animent des émissions soit au terme ou au début de leur carrière de chanteur. Nommons Hector Pellerin, Conrad Gauthier, dans les années 1920 [ce dernier fit sa première apparition au poste montréalais CHLP], Félix Leclerc ${ }^{11}$, Jean-Pierre Ferland en 1959 et Roland Lebrun.

On entend par animateur celui qui présente un spectacle en direct, qui présente les artistes ou fait lui-même un numéro. Ce sera le cas de quelques chanteurs tel Jacques Normand. C'est à Jean Lalonde que revient la tâche d'animer le «Quart d'heure de la Bonne Chanson » sur les ondes de la radio culturelle CKAC dans les années 1930 et 1940.

\section{Vulgarisation et diffusion des valeurs religieuses}

Le baby-boom de l'après-guerre associé à l'élévation du niveau de scolarité, suscite la création de collections éditoriales pour la jeunesse et la réédition notamment de romans historiques. La littérature et la radio sont des véhicules idéaux pour la transmission des valeurs chrétiennes chez les jeunes. Dans ce cadre, il est normal que la radio devienne aussi le support privilégié utilisé par des historiens comme Lionel Groulx, qui est présent à l'émission l'Heure provinciale à CKAC en 1929-1930 et qui diffuse au début des années 1950 son Histoire du Canada français avant de la publier. Dans la même période, l'abbé Charles-Emile Gadbois, directeur de l'entreprise éditoriale La Bonne Chanson, fonde la station CJMS (1954) (acronyme de Canada Je me souviens) afin de diffuser le répertoire de La Bonne Chanson tout comme le Quatuor Alouette qui lance l'émission le "Réveil rural », titre d'une chanson d'Alfred Desrochers qui ouvre l'émission (le générique). Mentionnons aussi l'action des docteurs Léo Pariseau et Ernest Gendron qui doivent, « afin de dissiper la méfiance qui persiste à l'égard de la science et [afin] d'assurer l'essor d'un enseignement scientifique [...], consacrer une partie importante de leurs activités à des tâches dites de vulgarisation » (Descarries-Bélanger et al. 15). C'est aussi la voie que choisira Fernand Seguin qui reçoit dans le cadre de son émission des chansonniers comme Gilles Vigneault. On lui connaît aussi une participation notoire à l'évolution de la programmation radiophonique dans le cadre de l'émission Carte Blanche où il présente notamment la causerie « La science en pantoufles ». La vulgarisation constitue une facette de la radio éducative et de ce que Lamizet et Silem appellent la 'communication médiatée', c'est-à-dire celle qui opère un renforcement du lien social en faisant apparaître l'importance de ce lieu (245). Différents événements comme le succès du feuilleton télévisé La famille Plouffe, ${ }^{12}$ diffusé sur les ondes de Radio-Canada entre 1953 et 1957, témoignent des mutations de la sensibilité et des mentalités à l'époque tout autant qu'ils les consolident (Létourneau 72). 
Les ondes radiophoniques des années 1940 font entendre les crooners américains (premières victimes d'érotomanie comme c'est le cas de Frank Sinatra) mais aussi les auteurs-compositeurs-interprètes ou interprètes français comme Charles Trenet, Georges Guétary ou Edith Piaf ${ }^{13}$. Non seulement les chanteurs interprètent-ils directement encore, comme dans les années 1920, leur répertoire sur les ondes mais en outre, ils continuent toujours d'animer leur propre émission perpétuant ainsi la fonction ce qui leur confère le statut de médiateur de l'activité chansonnière. Paul Brunelle animait d'ailleurs une émission à CKAC alors que Marcel Martel en animait une de 1945 à 1955 à CKVL.

Le chanteur folklorique Raoul Roy chante le répertoire des Archives de folklore sur les ondes de Radio-Canada International, entre autres. Plusieurs chanteurs y auront leur quart d'heure, dont Jacques Aubert et Lionel Parent. En 1956, CHLT ouvre ses portes. René Charest affirme que l'émission de Ti-Blanc Richard constitue une espèce de rampe de lancement : "Son émission est très écoutée et permet la découverte de plusieurs jeunes vedettes locales : les plus connues sont Michèle Richard.la fille de Ti-Blanc), Jacques Michel et son groupe Les colibris, Donald Lautrec et Jenny Rock » (180). Ti-Blanc Richard d'abord, et Marcel Martel ensuite (à partir de 1959), s'intègrent à la programmation. Les chanteurs de charme comme Jean Lalonde et Fernand Robidoux profitent aussi de la radio pour se faire connaître. Gemma Barra devient quant à elle vers 1956, l'une des premières chansonnières à avoir une émission régulière à la radio dans laquelle elle interprète ses propres compositions.

Ce n'est en fait qu'avec les années 1950, que la radio devient une rampe de lancement pour la chanson canadienne ainsi qu'une manière de freiner l'envahissement de la chanson étatsunienne. Ainsi, mentionne Chantal Hébert, l'oeuvre de Jacques Normand, plutôt orientée vers la promotion de la chanson française,

fait voir qu'à cette époque certains artistes menaient une double carrière, s'inspirant du travail qu'ils accomplissaient à la radio et qui assurait une certaine popularité pour mettre au point une revue ou leur numéro de cabaret. Le succès du «Faisan doré » par exemple, reposait póur une bonne part sur un mélange bien dosé de la formule des "chansonniers de Montmartre", de la chanson populaire et de la participation du public qui avait fait la fortune d'émissions comme le "Fantôme au clavier" et "la Parade de la chansonnette française" diffusées sur les ondes de CKVL. (603)

L'émission Baptiste et Marianne, animée par Guy Mauffette dans les années 1950, est un lieu privilégié pour stimuler la carrière de chanteurs ou de chansonniers qui veulent se faire connaître. Elle accueillit et fit découvrir Félix Leclerc ${ }^{14}$, ainsi que Guy Bélanger et Fernand Robidoux. Quant à ce dernier, il commence sa carrière en 1938 à CHLC en tant que chanteur, annonceur et technicien. Il animera aussi une émission à CHLN et à CHLP 
ainsi que la fameuse émission Ici Fernand Robidoux à CKAC qui fait aussi connaître les nouveaux noms de la chanson canadienne-française comme Raymond Lévesque (L'Herbier 63) dont il sera d'ailleurs le premier à interpréter les chansons. Robidoux luttera, en compagnie de l'animateurchanteur Robert L'Herbier, contre l'envahissement de la chanson étatsunienne sur les ondes de CHLC (Sherbrooke), de CHLN (TroisRivières), de CKVL (Verdun) et de CKAC (Montréal) (Chamberland et Gaulin 24). Parmi ceux qui gravitent autour de lui vers 1950, nommons les chansonniers Raymond Lévesque et Jacques Blanchet. Lévesque débute dans la série d'Henri Deyglun « Grand-Maman Marie » à CBF (L'Herbier 91 , Rivière 23) pour laquelle Henri Deyglun écrit les textes. R. Lévesque coanimait avec Serge Deyglun et Jeanne Maubourg, et d'autres chantaient leurs chansons. Il faut voir dans cette émission à laquelle participeront de nombreux auteurs-compositeurs, une espèce de laboratoire de la chanson québécoise pour reprendre l'expression de Robert Giroux et al. (31). De concert avec les écrivains, les chansonniers travaillent pour de nouveaux modes de communication (radio, télévision, etc.) et restent parfois, comme c'est le cas de Robidoux, au premier plan de la lutte pour une chanson d'expression française. Tex Lecor anime dans les années 1970 (Mon pays, mes chansons, etc.) et Pierre Létourneau (La boîte à chansons) en 1964 à Radio-Canada.

L'apparition de la télévision (en 1952) offrira, comme le souligne Robert Giroux, « une autre tribune de choix à la nouvelle chanson québécoise; Robert L'Herbier et Rollande Désormeaux, Raymond Lévesque (Paulette et Raymond avec Paulette de Courval) et Colette Bonheur animeront des émissions qui contribuent à susciter de nouveaux auteurs-compositeurs " (28). L'émission Rollande et Robert ouvre la voie au Concours de la chanson canadienne qui fera connaître, entre autres, Marc Gélinas. Ce concours, repris sous le nom la Chansons sur mesure en 1961, est lancé par Radio-Canada en 1956. Douze chansons seront primées le 22 février 1957. Dans les années 1980, des concours sont organisés par différentes stations de radio telle CKOI, qui parraine l'Empire des futures stars, et Radio-Canada Rock-envol, de même que la station FM 93 qui fut, grâce à Jacques Roy, un tremplin pour la promotion de jeunes groupes de Québec même si le CRTC avait sévèrement puni la station à la suite d'écoutes faites en $1983^{15}$.

Le parallèle que j'établis entre les émissions de radio animées par les chanteurs et les concours de chansons, provenant du fait que tous deux servent de rampes de lancement des chanteurs, $n$ 'est pas inintéressant car il montre les mécanismes de l'industrie radiophonique. Non seulement celle-ci sert-elle à la promotion du disque mais elle conduit aussi à la carrière discographique. Ce fait est peut-être plus notoire pour la tradition commerciale que pour la tradition savante. Le répertoire radiophonique s'est toujours partagé, comme le disque d'ailleurs, entre une tradition 
bourgeoise consommatrice de musique dite savante et la masse qui s'intéressait davantage au répertoire dit populaire, qu'il soit traditionnel ou commercial selon les deux acceptions du terme. Le coût élevé des disques favorise l'enregistrement du répertoire classique. Le parallèle entre la radio et le disque est notable parce que c'est le faible nombre d'enregistrements qui oblige les chanteurs à se produire en direct des studios (Du Berger et al. 82). Parfois les enregistrements d'émissions sont réalisés pour des réseaux nationaux. C'est le cas du "Quart d'heure de la Bonne Chanson », d'abord diffusé à CBF et ensuite à CKAC jusqu'en 1953, qui enregistre en direct des artistes comme Albert Viau et François Brunet, et qui est ensuite rediffusé sur le réseau national. Les réseaux reflètent les goûts des consommateurs et les activités radiophoniques de la Bonne Chanson, sous la tutelle de l'abbé Charles-Emile Gadbois, sont même adaptées pour les enfants et pour les adultes. On peut sans nul doute affirmer que la distribution des activités de consommation du disque se reflète aussi dans l'activité radiophonique. Ainsi, CHRC et CKCV étaient deux postes franchement distincts. CHRC était considéré comme «le poste de la famille et de l'information». Ce poste présente, commé CKAC, l'émission Chantons en cceur avec Woodhouse et soyons heureux et en tire une brochure publiée par Le Soleil pour Woodhouse. Le poste CKCV peut être qualifié de « station plus légère, plus jeune et plus locale» (37), connu entre autres pour ses concours (112). Le clergé trouvera sa place au sein d'un média (pensons à CJMS fondé par Raoul Gadbois, le frère de Charles-Emile Gadbois) qu'il trouve par ailleurs trop intéressé au moment présent parce qu'il constituait un mode de consommation culturelle « qui s'accordait bien mal avec le discours traditionnel » (Berger et al. 276).

\section{Conclusion}

Ce survol de l'histoire de la naissance de la télégraphie sans fil, de la radio éducative et des parallèles entre l'industrie du disque, de la chanson et de la radio a cherché à expliquer l'évolution complexe de la carrière du chanteur, véritable médiateur de l'activité chansonnière, au fil des décennies dans un contexte marqué par des changements techniques et économiques importants. La radio a ainsi souvent été, pour nombre d'entre eux, un tremplin pour l'activité chansonnière. Elle constituait, pour le chanteur, une façon de mieux accéder à la célébrité et elle s'offrait plus comme un médium de son répertoire qu'une manière d'exercer ses talents d'animateur. Le parcours du cabaretier et chanteur Jacques Normand est à ce titre exceptionnel. Propriétaire du Faisan Doré, il y invite les grands artistes des années 1940 qui se rencontraient parfois entre amis. Le Faisan Doré devient non seulement le foyer de passage pour un nombre élevé d'artistes français et québécois mais aussi la scène sur laquelle Normand pratique son répertoire. La scène du Faisan Doré ou d'autres des nuits de Montréal et de Québec, constituent un réseau parallèle que la radio sait alimenter par des programmations nationales. La radio a ainsi offert une 
tribune à des chanteurs en herbe comme Félix Leclerc, des artistes confirmés comme Jean Lalonde, des artistes partiellement retraités, du moins dans le domaine de la chanson, comme Raoul Duguay et aussi à des personnages littéraires comme Jean Narrache, pseudonyme d'Émille Coderre. Nous pensons à l'émission " Le Quart d'heure de la Bonne Chanson " qui tenta d'inculquer les valeurs traditionnelles par l'apprentissage de la chanson éducative moralisatrice intériorisée, les activités de Fernand Robidoux à qui la radio donna une forte visibilité, etc. Aussi, la radio servit de studio de répétition pour Charles Aznavour et Pierre Roche en 1948, deux interprètes au programme Jeunesse sur les ondes de CHRC (Du Berger et al. 84). La radio marque une certaine professionnalisation du métier de chanteur car les engagements comme artiste, chanteur, musicien ou comédien se faisaient par audition comme le souligne Jean Du Berger et al. (60).

L'étude des rapports entre la chanson et la radio constitue un enjeu majeur au sein des études en musique populaire si l'on connaît, par exemple, la fertilité des réflexions sur les palmarès, la distribution, les cotes d'écoute, les créneaux et les quotas à respecter (voir en particulier les travaux de Roger Chamberland et Robert Thérien à ce sujet). Ces engagements font voir l'importance que l'industrie radiophonique (en particulier CHRC et CKAC) a accordée, non sans heurts, à la promotion des chanteurs d'ici (comme Marius Delisle) sinon de la langue française. La rivalité interurbaine qui existe entre Québec et Montréal, connue dans le milieu littéraire depuis L'École littéraire de Montréal d'Octave Crémazie et l'École Patriotique de Québec d'Henri-Raymond Casgrain, connaît aussi certains épisodes dans le milieu radiophonique. Ainsi, Du Berger et al. affirment : « Quand ils commençaient à être expérimentés, le poste les perdait au profit de Montréal. Ce fut le cas des Pierre Marcotte, Miville Couture, Félix Leclerc [...]» (108).

Cela étant dit, la mobilité géographique et sociale des artistes chanteurs mériterait d'être approfondie, ne serait-ce que pour mieux comprendre les interactions qui ont toujours uni les acteurs du champ radiophonique et ceux de l'activité chansonnière. Aujourd'hui, souligne Roger Chamberland (110), de toutes les grandes villes nord-américaines, seule Montréal n'a pas de station country-western alors que pour le Québec tout entier, seules deux stations possèdent en 1996 un permis de diffusion de countrywestern.

En terminant, il convient de mentionner que l'influence de la musique traditionnelle sur la musique country américaine permet d'offrir au public qui commence à se désintéresser de la musique traditionnelle, une transition entre l'espace privée de la musique traditionnelle et l'espace publique qui sera celui de la musique country. Ainsi l'activité chansonnière country est très riche sur les ondes des radios comme sur scène à partir des 
années 1940, permettant ainsi de faire la transition entre une société marquée par la tradition orale et la commercialisation progressive de la chanson signée.

\section{Notes}

1. J'ai déjà abordé le sujet dans « Du journaliste au parolier : des multiples médiations de l'édition du texte chansonnier ", dans Regards sur la littérature québécoise, Hommage à Gaston Miron, Actes du troisième colloque des jeunes chercheurs européens en littérature québécoise [Gênes, 13 et 14 mai 1999], Hélène Amrit et Anna Giauffret-Harvey, Sergio Zoppi (a cura di), Roma (Italie), Bulzone Editore, 2001, p. 219-232.

2. La Canadian Marconi n'était qu'une filiale parmi plusieurs autres de la compagnie-mère fondée en Grande-Bretagne. La Marconi Wireless Company était basée à Londres et ne dirigeait pas comme telle ses filiales internationales.

3. La publicité est enseignée au HEC dès 1910.

4. Selon Thérien, Lionel Parent, étudiant à Nicolet, était probablement le premier chanteur canadien à se produire à la radio (106).

5. Bien que nous avons au Québec gardé à la mémoire surtout la pratique de chansons par des hommes, n'oublions pas que ce sont des femmes qui ont inauguré la pratique et que certains, dont Rudy Vallee, ont utilisé le porte-voix pour obtenir le même effet que le microphone plus tard. (Voir entre autres Bruce Johnson.)

6. Trad. «Avec l'avènement de la radio en particulier, les auditeurs pour un artiste précis ou pour une pièce de musique devinrent plus significatives et plus dispersées. Même si certains " stars " comme Al Jolson ont indubitablement existé avant l'avènement de la radio, l'enregistrement et le film créèrent une plus grande distance entre les artistes et leur public de masse et donnèrent alors place à un espace dans lequel cela devient plus facile de créer le phénomène de star. Les nouveaux média de la radio, du film, et plus tard de la télévision, auxquels s'ajoutent l'invention du phonographe et l'importance croissante de Muzak signifiaient que les goût du public pouvaient être crées autant dans l'espace public que dans l'espace privé (dans la chambre à coucher, au travail et même au supermarché), sans qu'on soit physiquement présent au spectacle. "

7. C'est aussi le cas de nombreuses radios dans le monde comme la Vlaams Radio Orkest créée en 1935 à Bruxelles.

8. En moins d'un an, trente-neuf permis furent accordés au Canada et, en 1924, le Québec compte six permis d'exploitation.

9. Trad.: « Quand nous pouvons entendre, à l'aide d'un phonographe, le créateur de Falstaff et d'Otello, nous savons que nous apprécions des interprétations immortelles dans leur concepts parce qu'elles ont été formées sous la supervision rigoureuse du compositeur lui-même. "

10. Notons aussi l'émission "Chansonniers canadiens " mise en onde par Guy Bélanger entre 1950 et 1956.

11. Gaulin mentionne que Leclerc, le chansonnier, fait ses débuts en 1946 avec son émission Théâtre dans ma guitare diffusée à la radio de Radio-Canada. Dès 1934, Leclerc est annonceur à CHRC, puis en 1938 à CHLN (6).

12. Feuilleton basé sur le roman de Roger Lemelin, Les Plouffe (1948), lequel compte parmi les romans peu nombreux évoquant le thème de la guerre. 
13. Nous voudrions évoquer, mais sans pouvoir aller plus dans le détail, les réactions du clergé face à la pénétration de la radio américaine dans la vie catholique. Mentionnons, dans les années 1930 , le mouvement de pression de la Ligue canadienne pour la radio. On lira avec intérêt les contributions de Marc Raboy, Jean-Guy Lacroix dans l'ouvrage sous la direction de Florian Sauvageau (1999).

14. Notons aussi son émission le Cabaret du soir qui penche, consacrée à la chanson à texte et à la poésie chantée diffusée le dimanche soir de 21 heures à minuit.

15. Il est à noter qu'il n'est pas impossible, mais seule une enquête approfondie pourrait le déterminer, qu'un règlement de compte entre différents employés de la station de radio, des rivalités avec la station CFLS de Lévis, et des activités illégales d'écoutes téléphoniques sur les employés aient servi les intérêts de certains membres du gouvernement ou de l'administration du Québec. Le dossier de presse ne faisait pas état de manière détaillée desdites opérations et rivalités qui ont eu lieu au moment où nous fréquentions régulièrement la station de radio en 1982,1983 et 1984.

\section{Bibliographie}

Albert, P., « Evolution des médias », dans Lucien Sfez, Dictionnaire critique de la communication, t. 2, Les Grands domaines d'application, Paris, PUF, 1993, p. 962-965.

[Anonyme], « Statistiques intéressantes », Passe-temps, 15 mai 1920, p. 236.

Bauer, Robert, The New Catalogue of Historical records (1898-1908/09), London, Sidwick and Jackson LTD, 1947 [1937 ], 494 p.

Baulu, Roger, CKAC, Une histoire d'amour, l'histoire magnifique du pionnier des ondes radiophoniques françaises dans le monde, avec la coll. de Raymond Taillefer, préface de Jean Drapeau, Montréal, Editions Internationales Alain Stanké, $1982,174 \mathrm{p}$.

Beauregard, Claude, "La propagande de Vichy et la réponse du gouvernement canadien ", Bulletin d'histoire politique, vol. $7 \mathrm{n}^{\circ} 2$, hiver 1999, p. 33-42.

Bellemare, Luc, [compte-rendu de] Jean-Nicolas De Surmont. 2001. La bonne chanson : Le commerce de la tradition en France et au Québec dans la première moitié du XX siècle, Montréal : Triptyque, 220 p. ISBN 2-89031-396-4 (couverture souple), Canadian University music Review, Revue de musique des universités canadiennes, vol. 24, $\mathrm{n}^{\circ} 1,2003, \mathrm{p} .107-112$.

Benoit, Réal, Madame Bolduc, préface de Doris Lussier, Montréal, les Éditions de l'Homme, 1959, $123 \mathrm{p}$.

Buckley, David and John Shepperd, Audience, Continuum Encyclopedia of Popular Music of the World, vol. 1, (ed.) John Shepherd, David Horn, Dave Laing, Paul Oliver and Peter Wicke, 2003, p. 158-164.

Chamberland Roger et André Gaulin avec la collaboration de Claude Légaré, Pierre G. Mailhot et Richard Plamondon, La chanson québécoise de la Bolduc à aujourd'hui, anthologie, Québec, Nuit Blanche éditeur, 1994, 593 p.

Charest, René, «La chanson populaire en Estrie » dans Robert Giroux (sous la direction de), la Chanson en question, Montréal, Triptyque, 1985, p. 177-194.

Coats, Darby, Canada's 50 Years of Broadcasting and Stories Stations Tell: Featuring the Pioneer Station XWA later given the call letters CFCF 1919-1969, Montréal, Canadian Marconi Co, [s. d.].

Cupido, Robert, « The Medium, the Message and the Modern : The Jubille Broadcast of $1927 »$, International Journal of Canadian Studies/Revue international des études canadiennes, $\mathrm{n}^{\circ} 26$, Fall/Automne 2002, p. 100-123.

De Surmont, Jean-Nicolas,(2005a), «Marchand, Charles », Dictionnaire biographique $d u$ Canada, t. 18, 1921-1930, Québec, Presses de l'Université Laval, 2005, p. 777-778.

— - « Les interactions entre l'interprète et son répertoire : de l'interprèteartisan à l'interprète-médiatisé ", Rabaska, revue d'ethnologie de.l'Amérique française, Société québécoise d'Ethnologie, Québec, n 3, 2005, p. 49-57. 
, [recension de] Propagande de guerre et nationalisme dans le radio-feuilleton, essai de Renée Legris avec la collaboration de Michel de Smet, Marie-Christine Leduc et Pierre Pagé, dans Dictionnaire de ouvres littéraires du Québec, Montréal, Fides, 2003, p. 763-764.

, « Du journaliste au parolier : des multiples médiations de l'édition du texte chansonnier ", dans Regards sur la littérature québécoise, Hommage à Gaston Miron, Actes du troisième colloque des jeunes chercheurs européens en littérature québécoise [Gênes, 13 et 14 mai 1999], Hélène Amrit et Anna Giauffret-Harvey, Sergio Zoppi (a cura di), Roma (Italie), Bulzone Editore, 2001, p. 219-232.

De Luca, Giuseppe « Foreword » daris Julian Morton Moses, Collectors' guide to American recording 1895-1925, foreward by Giuseppe De Luca, New-York, American Record Collectors exchange, 1949, $199 \mathrm{p}$.

Descarries-Bélanger, Francine et al., "Le Frère Marie-Victorin et les « Petites sciences », Recherches sociographiques, vol. XX n $1,1979$.

Du Berger, Jean, Jacques Mathieu [et] Martine Roberge, la Radio à Québec 1920-1960, Québec, Les Presses de l'Université Laval, 1997, 297 p.

Dubreuil, Stéphane, -CQD-, Toujours à l'écoute, Histoire de la radio maritime au Canada, Ottawa, Ministre des Travaux Publics et Services gouvernementaux Canada, $1998,140 \mathrm{p}$.

Fabbri, Franco, "La chanson ", dans Jean-Jacques Nattiez (sous la direction de), Musiques une encyclopédie pour le XXI siècle, Musiques du XX $X^{e}$ siècle avec la collaboration de Margaret Bent, Rossana Dalmonte et Mario Baroni, 2003, p. 674-702.

Gendron, Jean-Denis, « L'accent dit provincial du français parlé au Canada au XIX et $\mathrm{XX}^{\mathrm{e}}$ siècles : le témoignage des voyageurs ", dans Français du Canaida-Français de France VI Actes du sixième Colloque international d'Orford, du 26 au 29 septembre 2000, Edité par Louis Mercier avec la collaboration d'Hélène Cajolet-Laganière Tübingen, Max Niemeyer Verlag, 2004, p. 175-186.

Giroux, Robert, avec la collaboration de Constance Havard et Rock Lapalme, le Guide de la chanson québécoise, Montréal, Triptyque, Paris, Syros, Alternatives, 1991, $179 \mathrm{p}$.

Hébert, Chantal, «Nuit de Montréal», dans Maurice Lemire [et al.], Dictionnaire des ceuvres littéraires du Québec, t. 5, Montréal, Fides, 1984, p. 603.

Hébert, Pierre, "Chapitre XI Le contrôle du livre et de la lecture ", dans Jacques Michon (ed.) et al., Histoire de l'édition littéraire au Québec, vol. 2 , « Le temps des éditeurs ", Montréal, Fides, 2004, p. 389-414.

Johnson, Bruce, The Inaudible Music : Jazz, Gender and Australian Modernity, Sydney: Currency Press, 2000. 244 p.

Kallmann, Helmut, A History of music in Canada 1534-1914, foreword by Ernest Macmillan, Toronto, University of Toronto Press, 1981,317 p. [Edition originale 1960].

Krugler, David F., chap. 1 "Broadcasting without a License : the origins of the Voice of America" dans The Voice of America, The Domestic Propaganda Battles, 1945-1953, Columbia et Londres, University of Missouri Press, 2000, p. 12-51.

Labbé, Gabriel, les Pionniers du disque folklorique québécois, 1920-1950, préface d'Ovila Légaré, [Montréal], l'Aurore, 1977,216 p.

Lamizet, Bernard et Ahmed Silem (sous la direction de), Dictionnaire encyclopédique des sciences de l'information et de la communication, Paris, Ellipses, 1997, 590 p.

Lamonde, Yvan, Histoire sociale des idées au Québec 1896-1929, Montréal, Fides, $2004,330 \mathrm{p}$.

Laurence, Gérard, «Province de Québec » dans Hélène Eck, La guerre des ondes : Histoire des radios de langue française pendant la Deuxième Guerre mondiale, Montréal, Hurtibise $\mathrm{HMH}, 1985$.

Lavoie, Elzéar, «L'évolution de la radio au Canada français avant 1940», Recherches sociographiques, vol. XII, $\mathrm{n}^{\circ} 1$, Québec, Presses de l'Université Laval, janvier-avril 1971 , p. 17-49. 
, "La constitution d'une modernité culturelle populaire dans les médias au Québec (1900-1950)» dans Yvan Lamonde et Edith Trépanier (ed.), L'avènement de la modernité culturelle au Québec, Québec, IQRC, 1986, p. 253-298.

Leclerc, Félix, Tout Félix en chansons, établissement du texte Roger Chamberland, introduction d'André Gaulin, bibliographie et discographie Aurélien Boivin, Québec, Editions Nuit Blanche, 1996.

L'Écuyer, Sylvia, « La musique classique à la radio », dans Jean-Jacques Nattiez avec la collaboration de Margaret Bent, Rossana Dalmonte et Mario Baroni, Musiques une encyclopédie pour le $X X I^{e}$ siècle, Musiques du $X X^{e}$ siècle, Paris, Actes Sud/Cité de la musique, 2003, p. 954-968.

Létourneau, Jocelyn, Le Québec, les Québécois, Un parcours historique, [Québec], Musée de la Civilisation, 2004, Montréal, Fides, 127 p.

L'Herbier, Benoît, la Chanson québécoise; des origines à nos jours, Montréal, les Editions de l'Homme, 1974, 190 p.

MacMillan, Keith, "Radio diffusion 3 - les débuts de la radio au Canada ", Encyclopédie de la musique au Canada [dorénavant EMC], Montréal, Fides, 1983, p. 860.

Martel, Gaétan, «Il était une fois », La Scene Musicale, vol. 10 n 6, mars 2005, p. 63.

Miller, Mark, «Musique country 3-Rôle des médias», dans EMC, 1983, p. 691.

Moogk, Edward B., En remontant les années; l'histoire et l'héritage de l'enregistrement sonore au Canada des débuts à 1930, Ottawa, [s. é.], 1975, XII-447 p.

Nielsen, Greg Marc, Le Canada de Radio-Canada, Sociologie critique et Dialogisme culturel, Toronto, Editions du Gref, coll. Theoria ${ }^{\circ} 4,1994,205$ p.

Normand, Jacques, les Nuits de Montréal, préface de Roger Baulu et Charles Aznavour, Montréal, Éditions de la Presse, 1974, 191 p.

Pagé, Pierre, «Cinquante ans d'émissions religieuses à la radio québécoise, 1931-1983, De l'apologétique au dialogue avec les grandes religions », SCHEC, Etudes d'histoires religieuses, 68, 2002, p. 7-23.

- , «Introduction » dans Pierre Pagé avec la collaboration de Renée Legris, le Comique et l'humour à la radio québécoise. Aperçus historiques et textes choisis, 1930-1970, Montréal, les Éditions de la La Presse ltée, 1976, 677 p.

, Répertoire des cuvres de la littérature radiophonique québécoise 1930-1970, Montréal, Fides, 1975, 826 p.

Potvin, Gilles, «Montréal », Encyclopédie de la musique au Canada, Montréal, Fides, 1983 , p. 663.

Richard, Béatrice, La Mémoire de Dieppe, radioscopie d'un mythe, Montréal, VLB éditeur, 2002, $207 \mathrm{p}$.

Rivière, Sylvain (textes réunies par), Raymond Lévesque, on ne peut pas tout dire, Montréal, Editions Triptyque, 1997,269 p.

Saint-Amour, Robert, "Programmation radiophonique et chanson québécoise » dans Robert Giroux (sous la dir. de), la Chanson en question(s), Montréal, Editions Triptyque, 1985, p. 83-110.

Sauvageau, Florian, (sous la direction de), Variations sur l'influence culturelle américaine, Sainte-Foy, Les Presses de l'Université Laval, 1999, 262 p.

Sterling, Christopher, «Radio Broadcasting : Pre-1945», dans Daniel H. Johnston(ed), Encyclopedia of International media and Communications, Vol. 4 R-Z, Amsterdam et al., Academic Press, 2003, p. 13-23.

Thérien, Robert, L'histoire de l'enregistrement sonore au Québec et dans le monde 1878-1950, Québec, Université Laval, 2003, 233 p. 